\title{
Future Projections and Fallouts of Exponential Longevity and Revival from Cryopreservation
}

\author{
Vinod Nikhra* \\ Department of Medicine, India \\ *Corresponding author: Vinod Nikhra, Senior Chief Medical Officer and Consultant, Department of Medicine, , New Delhi, India
}

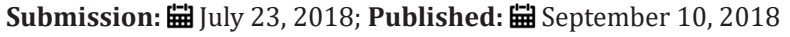

\begin{abstract}
Human lifespan and longevity: The aging is a universal phenomenon. With time, all life-forms age. In essence, the aging is a disorder of accumulated recurrent and chronic injury at cellular and molecular levels. It is influenced by the developmental damage load, harmful effects of early life situations, and adverse effects of chronic disease processes. The healthcare and healthy lifestyle help in slowing the aging process. The longer life and aging slowly are mutually related. There is nothing inherent in human constitution that limits the lifespan. At the biological level, evolution has prepared us for longevity, not the brevity.
\end{abstract}

Metabolic and genetic alterations: The metabolic dysfunction is linked to accelerated aging through generating free radicals that damage DNA and proteins. The aging may mean decay as the repairing process falters and there is a failure to restore and rejuvenate body tissues. The life span has also been linked to metabolic rate and metabolic stability. The genetic mutations in mitochondria trigger changes leading to apoptosis and accelerate the aging process. There have been discovered a variety of longevity mutations, which have been shown to extend life. Further, the genetic manipulations work through a common pathway across many species endorsing that there is an evolutionary genetic program that controls aging.

Caloric restriction for slowing aging: The state of nutrition has a bearing on aging and the life span. The CR protects against disease, slows aging and prolongs life. With CR regimen, there are less free radical production, less mitochondrial mutations and less programmed cell deaths. An optimal CR and CRAN reduce the incidence of virtually all diseases of aging such as cancer, CVD, diabetes, osteoporosis, auto-immune disorders, cognitive decline and diseases such as Alzheimer's and Parkinson's diseases, extend maximum and average life spans and improve disease resistance. Ingestion of fewer calories also alter favourably the fat deposition, adiposity and hormonal imbalance.

The cryonics and cryopreservation: Cryonics are the practice of freezing a body immediately after clinical death with the aim of enabling eventual revival in the remote future. The cryopreservation which is based on a more sophisticated understanding of death called 'information-theoretic death', offers a bridge to the life in future. The cryonics corporations have shifted emphasis from whole body to 'neuro-preservation' with the assumption that the rest of the body could be regrown and reconstructed in future. The main goal now is to preserve the information contained in the structure of the brain, on which memory and personal identity depends. The available scientific evidence suggests that the mechanical structure of the brain called connectome, is wholly responsible for personal identity and memories.

Conclusion: Futuristic visions of extreme survival-The Life extension program (LEP) aims to increase the maximum lifespan beyond the current maximum for humans. It can be visualised to go through three steps - taking advantage of the existing knowledge for slowing aging like CR and CRAN; utilizing the advances in genetics and biotechnology; and using the future nanotechnology and artificial intelligence revolution to repair the mutations and other defects due to aging at molecular and cellular levels. The futuristic goal is, thus, to achieve rejuvenation and state of non-aging, and to realize the eternal dream of exponential healthy life extension. The longer life is not separate from aging slowly, rather they are mutually related. With the scientific progress the futuristic visions of achieving significant longevity, if not immortality, seems quite possible. The eternal dream is on the verge of becoming a reality.

Abbreviations: CR: Caloric Restriction; CRAN: Caloric Restriction with Adequate Nutrition; CVD: Cardio-Vascular Disease; HBP: Human Brain Project; IGF: Insulin like Growth Factor; LEP: Life Extension Program; Mt-DNA: Mitochondrial-DNA; PNC-1: Pyrazinamide's and NiCotinamidase-1, QOL: Quality of Life; ROS: Reactive Oxygen Species; T2DM: Type 2 Diabetes Mellitus; SNP: Single Nucleotide Polymorphism; STAC: Sirtuin Activating Compounds

Keywords: Aging; Metabolic dysfunction; ROS; Longevity; Life extension; Cryonics; Human brain project; Caloric restriction; Human brain project; SIRT

\section{Human Lifespan and Longevity}

The aging is a universal phenomenon. With time, all life-forms age. The human-beings, too, age and grow older and afflicted with alterations at cellular and molecular levels in various body organs and tissues. The lifespan of organisms, including human beings, is not fixed but limited [1]. The incredible advances in the field of medical science have made possible to cure acute disorders and chronic diseases, and avoid frailty, complications and untimely demise. With improved lifestyles and better public health issues 
like sanitation and health care, there is an increased average life expectancy in general and improved survival rates in particular. The modern health-conscious man ages at a slower pace and lives a longer and healthy life. The slow aging and longer life are mutually related [2], and the concept of a healthy or successful aging can improve quality of life (QOL) and make longevity possible [3]. The aging issues concern the aging in terms of length of time as well as relate to the functional aspect with the older adults developing disability or incapacity for independent living as the result of damage load due to pre-existing diseases or due to the aging process itself.

As far as the aging research is concerned, there are three types of aging research. One, investigating aging the aging science researchers investigate and gather data, and do demographic studies of human longevity. Two, research for slowing the aging process - the effects of CR or the differences in life span between similar species and trying to reproduce some of those differences using drugs, gene therapies and other means. Three, the research for reversing aging - concerns ways to repair, reverse, or work around the root causes of aging. This last one is held as the only viable approach for producing rejuvenation biotechnology capable of greatly extending healthy life with youthful function and vigour.

\section{The Aging Process}

\section{Attrition and stress of living}

The aging is a disorder of accumulated recurrent and chronic injury at cellular and molecular levels [4]. The developmental damage load during early phases of life complicates further, having a bearing on the residual functional capacity and the aging process. The aging process is, thus, influenced by 3 factors: the developmental damage load, damaging effects of early life situations, and adverse effects of chronic disease processes. The optimal healthcare and healthy lifestyle help in slowing the aging process.

The three main causes of biological decay are disease, trauma and aging. The process of aging begins much earlier before becoming apparent and is a gradual collection of cumulative changes at the cellular and molecular levels and made of various components. There occurs exposure to continuous attrition and damage, and accumulation of wastes and toxins [5]. There occur some alterations in genome due to inner causes like mitochondrial generation of free radicals and external factors like cosmic radiations. Oxidative stress is also a potential source of damage at molecular level. Free radicals are generated within mitochondria and have potential to alter the cellular material and internal structures. These factors have been linked with aging process at the cellular level.

\section{Ros and metabolic dysfunction}

Under the socio-cultural layers, we human beings are biological animals and share the same fate as other members of the bio-spora. Several external, behavioural and internal factors including the environment, genes, sex and reproduction, nutrition and food style, the accumulation of cellular attrition and damage, and the inherent ability to repair, influence aging process, life span and QOL during lifespan. Whereas aging may mean decaying, as the repairing process weakens, the living means restoring and rejuvenating the body tissues [6]. At later phase in life, the aging system falters [7] and the aging process contributes to the age-related decline in performance, productivity and health. The cells possess a number of systems for functional and structural maintenance and repair. In fact, the DNA-repair capability correlates well with the lifespan of a species. Also, there is a correlation between the ability to respond to stress and life span [8]. The genes influence the life span of organisms, the body appearance, structure and functioning of inner organs, and account for about 25 percent of what determines the life span.

The free radical theory is fundamental to the understanding of aging [9]. The uncontrolled metabolic dysfunction is linked to accelerated aging. Thus, obesity is a disturbed metabolic state, having potential to cause metabolic syndrome, in which insulin resistance leads to diabetes, CVD and other related disorders. The life span has been linked to metabolic rate. The metabolism generates ROS that can damage DNA and proteins. Animals that live fast, die young; because a high metabolic rate produces large number of free radicals. According to this theory, long-lived animals should have high concentrations of antioxidant enzymes in their tissues and low concentrations of free radicals. Another related theory states that metabolic stability is a better predictor of longevity than metabolic rate. It proposes that an organism's ability to maintain stable levels of free radicals is more important than how fast it produces them. Further, metabolic stability is more important than metabolic rate in determining life span, and the long-lived organisms are more resistant to pathogens and other environmental stresses.

\section{The genetic influence}

The mitochondrial genetic mutations: The genetic mutations in mitochondria, appear to trigger apoptosis and accelerate the aging process [10]. Altering a gene called polymerase- $y$, which functions as a spellchecker during the copying of mitochondrial DNA in mice led them to age fast. Because the mitochondria also control the natural process of cell death, called apoptosis, mistakes by the spell-checker gene enhance apoptosis. As mitochondrial mutations accumulate, there are increased cell deaths and appearance of the aging characteristics.

The single gene mutations in humans: There have been discovered a variety of longevity mutations in species ranging from C. elegans to Drosophila to mammals. These mutations have been shown to extend life by about $50 \%$ in mammals and more in nematodes. In human longevity studies, single nucleotide polymorphism (SNP) analysis identified a large number of genetic variants with metabolic effects [11]. About a hundred of these metabolic manipulations enhance longevity by retarding aging comparable to $\mathrm{CR}$, are not as effective as repair strategies which can reverse aging process. The genetic manipulations causing these alterations work through a common pathway across many species endorsing that there is an evolutionary genetic program that controls aging. Further, it appears that the metabolic changes 
will not produce significant effects on longevity in humans as they do in mice for the simple reason that lifespan is much more plastic in short-lived species. Some of the important potential target genes for future gene therapies to retard aging or compensating for agerelated damage [12], are:

1) Angiotensin-converting enzyme (ACE): Lowered levels of ACE have been shown to extend mean life span in C Elegans.

2) Adenylyl Cyclase Type 5 (AC5): Knockout of AC5 extends life in mice possibly due to increased resilience of the cardiovascular system. Some aspects of AC5 knockout mice resemble those of CR mice.

3) AMPK: Targeted overexpression of AMPK in Drosophila increases life span. This has been related to CR response leading to improved cell maintenance and modestly slowed aging.

4) Angiopoietin-like 4 (ANGPTL4): The rare variant of this gene, present in less than $1 \%$ of the European population, reduces the risk of heart attack by half possibly due to alternations in cholesterol metabolism.

5) Angiotensin II receptor type 1 (Agtr1a): Lowering Agtr1a levels protects mitochondrial function and modestly extends life in mice.

6) Apolipoprotein A-1: Increased amounts of Apo A-1 improve cholesterol metabolism and slows progression of atherosclerosis by transporting away the damaged lipids build up in blood vessel walls.

7) $\quad 32$ microglobulin (B2M): B2M levels rise with age, and in mice reducing levels of $\mathrm{B} 2 \mathrm{M}$ in older mice restores some of the loss of cognitive decline due to aging. The role of B2M also relates to adaptive immune system.

8) C-Myc: Lowered levels of c-Myc retard aging and extend life in mice through its effects on insulin metabolism.

9) C1Q: The C1Q gene plays a role in the immune system. Its levels rise in brain with aging and removing it improves the state of cognitive function in older mice.

10) Catalase: Gene therapy to increase levels of the antioxidant catalase in the mitochondria reduces damage to mitochondria as a result of ROS.

11) CLK1: Reduced CLK1 activity extends life in mice due to altered mitochondrial function and consequently lowered generation of ROS.

12) Cyclin A2: The increased levels of cyclin A2 increase the regenerative capacity of heart tissue. It may also be beneficial in retarding degeneration in the heart.

13) FGF21: Overexpression of FGF21 occurs as CR response, and when induced using gene therapy can extend life in mice. The aging retarding effect is connected to growth hormone/insulin-like growth factor-1 signaling pathway.
14) Follistatin: Its increased level leads to muscle growth, a potentially useful compensation for the loss of muscle mass and strength that occurs with aging.

15) FOXO3: A variant of FOXO3 is associated with a modest reduction in CVD and mortality in human-beings.

16) GDF11: Higher levels of GDF11 improve various factors related to aging in mice, such as heart function and exercise capacity, possibly due to increased stem cell activity.

17) Growth hormone/growth hormone receptor/insulin-like growth factor/insulin receptor: Disrupting the growth hormoneinsulin metabolism extend life in mice.

18) Interleukin-21 (IL-21): The higher levels of IL-21 improve the state of the immune system by increasing the pace at which new immune cells are generated. May correct the loss of immune function with age.

19) KLF4: Selectively lowering levels of klf4 in improves smooth muscle cells function in blood vessel walls. Their overreaction to damaged lipids arriving in the bloodstream is muted, which slows the progression of damage and reaction to towards atherosclerosis.

20) miR-195: The microRNA miR-195 interacts with telomerase, and inhibiting it has much the same beneficial effect on stem activity as increasing levels of telomerase.

21) Mechanistic target of rapamycin (mTOR): Alterations to the mTOR gene have been shown to modestly extend life span in several species. The mTOR protein is involved in many fundamental cellular processes and cellular metabolism.

22) Myostatin: Reduced myostatin increases muscle growth, may be a useful for the loss of muscle mass with aging.

23) NF-кB: Its inhibition extends life modestly in a number of lower species, given its involvement in immunity, inflammation, apoptosis, and other fundamental processes.

24) P21: Both MRL mice and P21 knockout mice can regenerate small injuries with no scarring and reduced levels of the p21 protein seems to be the common factor in these engineered mouse lineages. P21 is closely related to the tumour suppressor gene P53.

25) P53: The protein $p 53$ plays the role of tumour suppressor. An increase in p53 levels accelerate aging by reducing tissue maintenance through the creation of new cells.

26) PCSK9: Loss of function mutations in PCSK9 reduce the risk of cardiovascular disease, most likely through lowered blood cholesterol levels.

27) plasminogen activator inhibitor-1 (PAI-1): Reducing levels of PAI-1 appears to modestly slow aging, possibly by removing one aspect of the harmful impact of senescent cells. 
28) Pregnancy-associated plasma protein-A (PAPP-A): Knockout of the PAPP-A gene interferes with insulin metabolism and produces a similar extension of health and life in mice when compared with other methods of achieving this end.

29) Phosphatase and Tensin homolog (PTEN): Adding an extra copy of the tumour suppressor gene PTEN to mice produces lower rates of cancer, much as expected, but also increased life span.

30) Rpd3: A reduction in Rpd3 level produces improved cardiac function and modestly increased longevity in flies.

31) TGF- $\beta 1$ : TGF- $\beta 1$ expression rises with age and is implicated in loss of stem cell function.

32) Troponin C: Researchers have shown that delivering a modified version of the calcium receptor troponin $\mathrm{C}$ into the mammalian heart can improve heart function and the performance of the cardiovascular system.

33) Uncoupling proteins (UCP): Uncoupling proteins manipulate mitochondrial function. The altered levels or genetic variants can improve health and longevity.

34) Urokinase (UPA): The $\alpha$ MUPA mouse lineage has the addition of a urokinase gene and has a longer life span. The UPA gene is related to PAI-1 and is argued to achieve life extension in mice through behavioural change - these mice eat less, and thus the CR response comes into play.

35) Telomerase: Increased levels of telomerase have been shown to extend life in mice, as well as reducing cancer incidence. The most possible mechanism appears to be increased stem cell activity, while effects on cancer may involve a more active immune system.

\section{Slowing Aging Process: CR and CR with Adequate Nutrition}

\section{Caloric restriction (CR)}

The state of nutrition influences the aging process and thus, the life span and life expectancy. In general, the CR regimen protects against diseases of aging and slows aging (Figure 1).

The CR is associated with lower levels of circulating blood glucose, insulin and triglycerides. Further, CR effectively reduces the free radicals production, mitochondrial mutations and programmed cell deaths. The effects of CR on aging process and lifespan are applicable to virtually all species. An optimal CR and CRAN reduce the incidence of virtually all diseases of aging such as cancer, CVD, diabetes, osteoporosis, auto-immune disorders, cognitive decline and diseases such as Alzheimer's and Parkinson's diseases, extend maximum and average life spans and improve disease resistance. Ingestion of fewer calories also alter fat deposition, adiposity and hormonal imbalance [13]. There are, though, certain adverse effects related to CR. General malaise, hunger pangs, a decreased ability to handle stresses, such as cold temperatures or infection, osteoporosis and loss of muscle mass, and loss of fertility can occur with un-supervised CR.

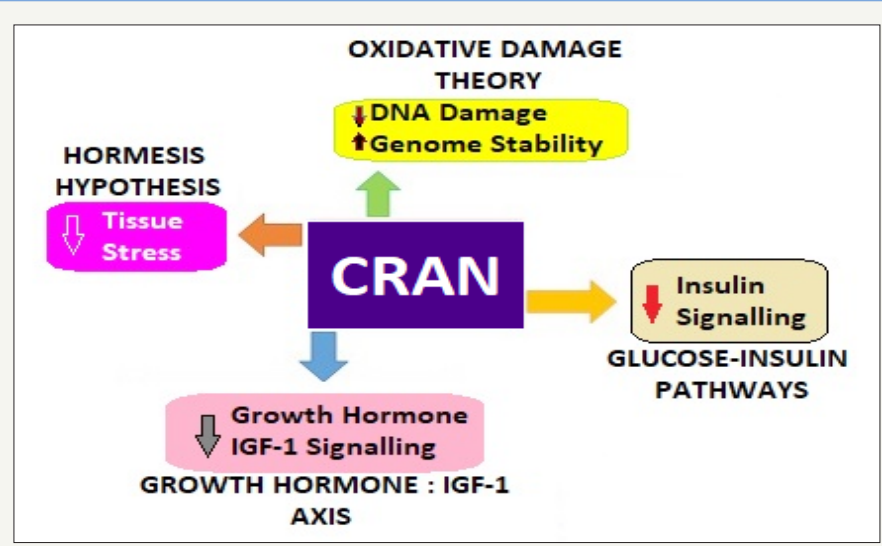

Figure 1: Caloric restriction and longevity circuits.

\section{The links between sirtuins and CR}

There exists an intricate relationship between sirtuins, insulinsignaling pathway and CR [14]. Both insulin and insulin-like growth factor-1 (IGF-1) affect sirtuin expression. In humans as in mammals, the SIRT1 gene (equivalent to Sir2 in yeast) rises with fall in insulin and IGF-1 levels and on CR. Sirtuins are also triggered by STACs, as with CR. Thus, STACs can get the benefits of CR [15]. The Sir2 gene acts to stabilize the chromosome, leading to increase in both the average and maximum lifespans increase (Figure 2). With an extra copy of Sir2 gene the yeast cells generate about twice as much sir2 protein and stabilizing the DNA, the yeast lived about 30 percent longer. The sirtuins are, thus, pro-survival molecules. Sir2, the member of a family of sirtuins genes, is present in all complex life forms. The STACs activate the sir2 protein. The STACs act through Sir2, and ineffective when the gene is deleted. The STACs have been found universally efficacious, in humans as well.

A gene, the master regulator, PNC1 controls Sir2. Stress turns on the PNC1 gene, the activity of which turns on Sir2. The adipose tissue is known to make hormones called adipokines, which may act on other tissues to alter longevity. The fat is also a source of 
molecules involved in oxidative stress, such as free radicals. Those on CR exhibit an altered metabolism and are slightly less efficient at converting food into energy but produce fewer free radicals and less oxidative damage. In human muscle, there is a decrease in oxidative metabolism with age. Relating to diabetes, oxidative metabolism and aging in muscle and fat, there appears to be a common oxidative pathway that becomes less efficient with age.

\section{Cryonics and Beyond: Cryofreezing for the Future}

\section{Defining clinical and terminal death}

Cryonics is the practice of freezing a body immediately after clinical death with the aim of enabling eventual revival in the remote future (Figure 3). The cryonics is, thus, a speculative life support technology that seeks to preserve human life in a state that will be viable and treatable by future medicine [16]. The cryopreservation which is based on a more sophisticated understanding of death called 'information-theoretic death', offers a bridge to the life in future [17]. The current medical and legal definition of death is based on the cardiac arrest and the cessation of electrical activity in the cerebral cortex. Whereas, a person is dead according to the information theoretic criterion if the memories, personality, hopes, dreams, etc. have been destroyed in the information theoretic sense, and the structures in the brain that encode memory and personality have been so disrupted that it is no longer possible to recover them. The information-theoretic death is 'absolutely irreversible death' and the destruction of the brain has occurred to such an extent that any information it may have ever held is irrevocably lost for all eternity [18].

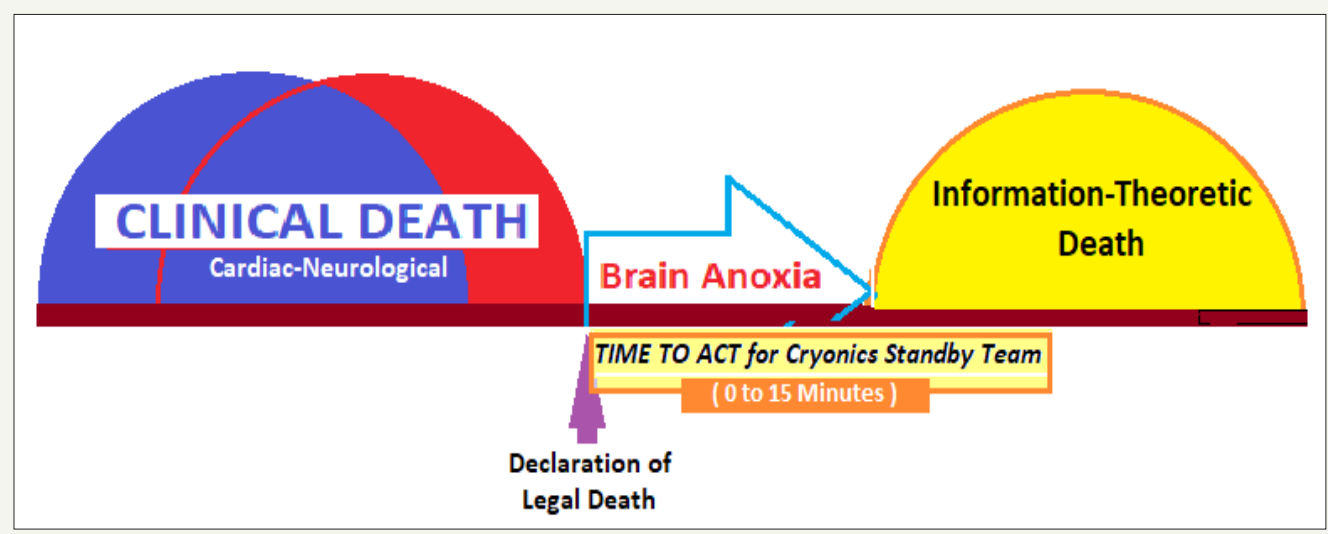

Figure 2: Concepts for cryonics: clinical-legal and information-theoretic death.

Legal death declared, process to begin in 2-15 min.
The body packed with Ice, and
Injected with chemicals to stop clotting

Figure 3: Cryopreservation in action.

Further, the cryo-preserved bodies are not irreversibly dead as per the information-theoretic definition of death. They are kept indefinitely preserved in a thermos-type container filled with liquid nitrogen until the cryo-preservation damage would be possibly reversed at some time in future when the advanced state of science and technology will allow the cause of the fatal disease to be cured and repair the damage to the body because of the aging process.

\section{Cryopreservation: The frozen life}

The cryopreservation process should start immediately after clinical-legal death is declared as organs remain biologically alive for some time, and vitrification, particularly of the brain, is possible. The clinical-legal death declaration indicates that there is nothing more can be done by the medical personnel to save the patient. of course, the declaration of death does not mean that life has 
suddenly ended-death is not a sudden event but a gradual process. The Stand-by Cryonics Team acts immediately, to minimize ischemic and reperfusion injury by beginning cardiopulmonary support and cooling as soon as possible after pronouncement of death (Figure 4), as the body is suitable for cryopreservation or the preservation of the brain for a short time only.

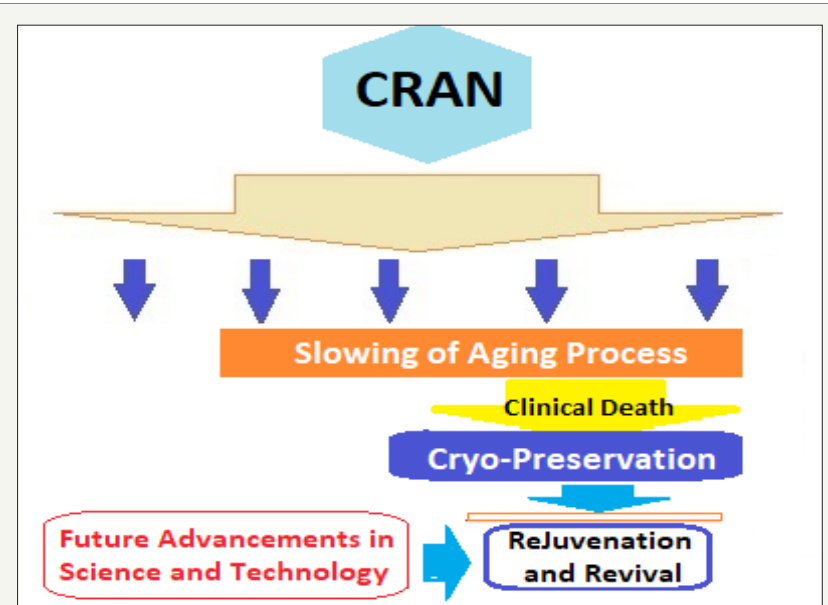

Figure 4: The future projections for exponential longevity, damage repair, rejuvenation and revival (CRAN: Caloric Restriction with Adequate Nutrition).

The body is cooled to just above $0{ }^{\circ} \mathrm{C}$ and the blood is replaced with a preservant and a solution is injected to stop ice crystals formation in organs and tissues. Thereafter, the body is further cooled gradually to $-130{ }^{\circ} \mathrm{C}$ and the anti-freeze compounds are injected to stop damage to the cells. The finally the body is placed in a container which is lowered into a tank of liquid nitrogen at -196 ${ }^{\circ} \mathrm{C}$. Alternatively, the head with brain inside is cryopreserved in liquid nitrogen at $-196^{\circ} \mathrm{C}$, contained in the Dewar flask, an insulated container which consumes no electric power. Newer forms of cryonics use a process called vitrification [19]. The vitrification employs low temperatures and cryoprotectants to turn tissue into a glass-like state where decay is extremely slow. It is also possible to develop hybrid procedures involving elements of both cryonics and chemical brain preservation [20].

\section{Head (neuro-) vs whole-body cropreservation}

During the 1980s, the cryonics corporations shifted emphasis from whole body to 'neuro-preservation' (i.e. head-only cryopreservation), with the assumption that the rest of the body could be regrown and reconstructed in future by nanobiotechnology. The main goal now is to preserve the information contained in the structure of the brain, on which memory and personal identity depends, and the available scientific and medical evidence suggests that the mechanical structure of the brain called connectome, is wholly responsible for personal identity and memories. The chemical brain preservation, viewed as a life-saving medical procedure, allows the brain to be preserved for a long period and in the future, the information in a chemically preserved brain may be able to be decoded and emulated in a computer. The main limitation of current cryonics is the uncertainty whether the information in the brain is truly preserved, though there is indirect evidence that cryonics preserves the information in the brain which could be recovered.
The hypothesis of chemical brain preservation as life extension was proposed by Drexler [21] and Olson in 1988. The brain is completely responsible for the mind and the identity is defined by anatomy: that is, brain connectivity. Our memories and personalities are captured in the synaptic and dendritic connections in the brain, referred to as the connectome [22,23]. The information theory of death and understanding of the connectome imply that death does not occur until the information in the connectome is irreversibly lost $[24,25]$. In the distant future, technology may advance to the state where the information of an individual's brain design can be extracted from the preserved brain and the complete connectome to be obtained from preserved brains, the whole brain emulation (WBE). Knowledge of the connectome should allow for a complete emulation of brain function, and the technologies for mapping the connectome and for WBE have been advancing rapidly [26]. The development of $\mathrm{WBE}$ and the computer technology to implement it is now an initiative of the European Union known as the Human Brain Project [27], which aims to develop a complete emulation of a mouse brain and later that for the human brain. The Human Brain Project aims to scan and upload a significant portion of the human brain [28].

\section{Human brain project}

The Human Brain Project (HBP) is a scientific research project that aims to build a collaborative information and communications technology (ICT)-based scientific research infrastructure to allow researchers across Europe to advance knowledge in the fields of neuroscience, computing, and brain-related medicine [29]. The Project, which started in 2013, is a European commission future and emerging technologies flagship. The HBP is largely funded by the European Union. The project is based in Geneva, Switzerland. The primary objective of the HBP is to create an ICT-based research infrastructure for brain research, cognitive neuroscience and brain- 
inspired computing, which can be used by researchers world-wide. The Human Brain Project (HBP) is building a research infrastructure to help advance neuroscience, medicine and computing.

Six ICT research platforms form the heart of the HBP infrastructure-Neuro-informatics (access to shared brain data), Brain simulation (replication of brain architecture and activity on computers), High performance analytics and computing (providing the required computing and analytics capabilities), Medical Informatics (access to patient data, identification of disease signatures), Neuromorphic Computing (development of braininspired computing) and Neurorobotics (use of robots to test brain simulations).

The HBP also undertakes targeted research and theoretical studies, and explores brain structure and function in humans, rodents and other species. The timeline of the Project is split into multiple phases, each of which will be covered by a separate funding agreement. The current phase is specific grant agreement two (SGA2), which spans the two-year period from April 2018-April 2020. The Human Brain Project strives to accelerate the fields of neuroscience, computing and brain-related medicine. This acceleration will be achieved by a strategic alignment of scientific research programmes in fundamental neuroscience, advanced simulation and multi-scale modelling with the construction of an enabling Research Infrastructure.

\section{Cryonics: The facts}

Around 350 people worldwide have had their bodies preserved in cryogenic chambers after death in the hope to be revived in the future. The experts at the Cryonics Institute (CI), Michigan have claimed that cryonically bringing someone back to life should definitely be doable in 100 years or sooner. The CI has about 160 patients frozen in specialised tanks of liquid nitrogen at its headquarters and has almost 2,000 people signed up to be frozen after they die [30]. Two main US cryonics organisations are Alcor at Arizona, and the CI at Michigan. A Russian body Krio Rus and Alcor's European laboratory in Portugal are the two facilities outside the US to offer the service. Aside from Trans Time, the other three cryonics organizations in the world which are storing human patients in liquid nitrogen are the Alcor Life Extension Foundation (founded in 1972 by Fred and Linda Chamberlain), the Cryonics Institute (founded in 1976 by Robert Ettinger), and Krio Rus (located near Moscow in Russia, founded in 2006). It is being claimed that cryonics may replace traditional burials and cremations in the next few decades, leading to Cryo-Parlours in place of Funeral Parlours.

Futuristic Visions of Longevity: Projections for Exponential Lifespan

\section{Anti-aging and rejuvenation science}

In the current times, there is an increased awareness for fitness and health, and enthusiasm for living a long life [31]. The latter is winning over the prejudices, fixations and obstacles, and exciting changes are taking place in the scientific world of gerontology, biotechnology and nanobiology which hold immense promise for slowing the aging and prolonging lifespan. The healthy aging puts forth three options before us. We can try to improve metabolism so that it generates less harmful by-products as by CR or we can clean these up these by-products, or deal with the consequences of the accumulation of damage over time.

Where do we stand on the issue of prolonging lifespan and what lies in store for us in the near and far future? What are the expectations from the aging research and the projections and fallouts? The futurist visionaries believe that it will be possible to maintain human health at a youthful level for many extra decades by healthy living, employing new methods of anti-aging medicine and rejuvenation science to retard and probably reverse the aging. Since aging is consequence the damage accumulation, repair of the damage forms the basis of rejuvenation, the reversal of aging and prevention of degenerative and age-related diseases. The regenerative therapies aim to remove, repair, replace or render harmless the cellular and molecular damage accumulated in tissues with time, and restore the normal functioning of the tissues and essential biomolecules.

Exponential life extension: The visions for future: The Life extension stands for an increase in the maximum lifespan beyond the current maximum lifespan for humans, and the exponential life extension can be defined as increase in life expectancy and life span by 50 per cent or more [32]. For those who regard aging as a disease, therapeutic methods to extend maximum lifespan are anti-aging medicine. The recent development in life extension is the vision that the damage to macromolecules, cells, tissues and organs can be repaired by advanced nanobiotechnology [33]. As we understand the biological principles of life and aging process, and able to utilize the experimental research, the life extension program (LEP) can be seen as a plausible step in near future. The LEP is projected to go through three steps - Step One: Taking advantage of the existing knowledge for slowing aging like CR; Step Two: Utilizing the advances in genetics and biotechnology; and Step Three: Using the future nanotechnology and artificial intelligence revolution to repair the mutations and other defects due to aging at molecular and cellular levels. The way to cure aging is to rejuvenate tissues, not just to retard aging. The futuristic goal, thus, is to achieve rejuvenation and the state of non-aging [34].

\section{THE aging-related cellular damage:}

First, the cell loss: Certain tissues, like heart and brain, lose cells with aging and these cells are not naturally replaced. The cells are damaged by both tiny molecular-level insults and by obvious trauma. Some of these damaged cells are repaired, but others are either destroyed, or forced into a dysfunctional 'senescent' state where they can no longer divide. Some of the lost cells are replaced by the pools of specialized, tissue-specific stem cells, but the degenerative aging process makes the stem cell pools less effective 
at repair over time. Thus, over the course of years, long-lived tissues like brain, heart, and skeletal muscles begin to progressively lose cells, and their function becomes increasingly compromised. The stem cell therapy and tissue engineering can be used to restore the cells in these tissues.

Second, mutations: Mutations in chromosomes affect organ function and the life span and can cause cancer. Two types of damage accumulate in genes with age: mutations and epimutations. Mutations are damage to the DNA sequence itself, whereas epimutations are damage to the scaffolding of that DNA, which controls how and when genes get turned on in the cell. Both mutations and epimutations ultimately harm by causing abnormal gene expression which causes cancer. A Targeted gene therapy can be used to delete the telomere elongation genes in particular tissues at risk of developing cancer.

Third, Mt-DNA injury: The mitochondria generate highlyreactive molecules, free radicals, which can damage cellular structures and its own DNA (Mt-DNA) which is especially vulnerable to these free radicals, being located so close to the centre of its production. A free radical hit to the mt-DNA can cause major mutations and deletions in its genetic code, eliminating the mitochondriass ability to use the instructions to make proteins that are critical components of their energy-generating system. The mutant mitochondria enter into an abnormal metabolic state that produces little energy, while generating large amounts of ROS. Certain forms of mitochondrial DNA damage in cells produce malfunctioning mitochondria and contributes to the progression of atherosclerosis via increased amounts of oxidized lipids in the bloodstream. The insertional gene therapy can introduce modified versions of the 13 protein-coding mitochondrial genes into nuclear DNA, which will prevent accumulation of mutations in the mitochondrial DNA.

Fourth, the senescent cells: The senescent cells halt growth, alter the supporting tissue architecture and secrete large amounts of inflammatory compounds. After decades of accumulation, their number becomes large and with their abnormal metabolic state pose a threat to surrounding healthy tissues. The approach to deal with these cells is to destroy them by a drug toxic to the senescent cells or Immune therapy which selectively destroys senescent cells.

Fifth, the extracellular cross-linking: Blood sugar and other molecules react with the proteins to create chemical bonds called crosslinks, which hamper with the normal functioning of various tissues. The constituent proteins require to maintain their proper structure and these proteins are responsible for the elasticity of the artery wall, the transparency of the lens of the eye, and the high tensile strength of the ligaments, etc. The crosslinked strands of collagen become over time make the blood vessels to become rigid and cause a gradual rise in systolic blood pressure with age. The cross-link-induced loss of elasticity in blood vessels having a major influence on mortality through hypertension and consequent impact on cardiovascular health and the loss of the cushioning effect provided by free-moving collagen lead to a surge in the vessels having a damaging effect on organs like kidneys and brain. The most significant contributor to the collagen crosslinking in humans is a glucosepane a complex molecule. The cross links between long-lived molecules in the extra-cellular matrix, such as collagen and elastin can be broken by designer drugs. Clearance of glucosepane cross-Links in the extracellular matrix of tissues is one of the early rejuvenation therapies.

Sixth, the extra cellular junk: This is important in Alzheimer's and Parkinson's diseases and other neurodegenerative disorders. A2E seems to be responsible for macular degeneration. Another form of extracellular junk, beta-amyloid forms plaques in the brains of Alzheimer's disease patients and impairs cognitive function. There are about twenty different types of amyloid, misfolded proteins that form solid deposits and are associated with agerelated dysfunction. The transthyretin amyloid is associated with heart disease and is thought to be the primary cause of death in supercentenarians. Amyloid- $\beta$ is one of the forms of metabolic waste that accumulates in Alzheimer's disease. There are other amyloid aggregates in other tissues during aging and contribute to age-related diseases, including islet amyloid in T2DM.

The needful therapy will slow down the accumulation and get rid of it as well. The insertional gene therapy by introducing bacterial or fungal genes can break down the damaging accumulated chemicals and proteins, including oxidized cholesterol causing atherosclerosis. The extracellular aggregates can be removed from the brain and other areas of the body by specialized antibodies. The active vaccines introduce a small fragment of the amyloid to stimulate the cells of the immune system to target the amyloid and remove it. The Passive vaccines involve making the antibodies outside of the body and introducing them directly via injection. A third and promising variation is a subset of human antibodies have catalytic activity against a particular antigen, breaking it down into smaller and less harmful fragments.

\section{The seventh, Miscellaneous:}

A. For the aging immune system: The thymic rejuvenation may increase the supply of immune cells. Another possible approach to partially restore lost function in the aging immune system is to increase the pace at which new immune cells are created. The thymus acts as a nursery for the maturation of $\mathrm{T}$ cells, and its atrophy thus restricts the rate at which new cells enter circulation. There has been some progress towards engineering of replacement active thymus tissue, as well as methods of providing signal proteins that instruct the old thymus to regenerate and begin to act in a more youthful manner. Transplants of young thymus organs into old mice has demonstrated that this class of approach can produce a meaningful improvement in immune function, and thereby extend healthy life.

B. To Reverse Stem Cell Aging: The stem cells aging is driven by the accumulation of waste products like glucosepane cross-links and the components of lipofuscin that degrade lysosomal function in long-lived cells, or because clearance systems fail over time, as 
appears likely to be the case for the amyloid. The removal of waste products from the stem cells environ will provide a solution.

Finally, to keep alive or preserved: Till the advanced technology is practically available. Until molecular repair technologies are available, good health practices, nutritional supplements, CR and organ transplantation, and cryopreservation after death are the best hopes.

The state of non-aging: concept of immortality: Immortality or eternal life is the concept of existing for a potentially infinite length of time. The ideas of immortality have existed in mythological tales and the sci-fi writers have also woven immortal worlds in their fiction. In more recent and current times, people have had their dead bodies cryopreserved, with the hope that scientific and technological advances will allow them to be unfrozen, cured, and restored to health at some point in the future [35]. The three possible futuristic outcomes may seem probable: The first, we will live and die as we do today. There may accrue no benefit of aging research. The second possibility, called the Tithonus option, is that the technology will give extended lifespan but will not be able to reduce prevalence of dementia and debility. The third possibility is that technology will be able to repair the damage done to our tissues with age, including neurons, thus granting us longevity with good quality of life.

There are three main causes of death: aging, disease and trauma. The hardest cause of death to overcome is trauma. In the postulated future world where aging will be correctable and diseases will be triumphed over, a trauma would still kill, unless the technology advances to such extent that a body can automatically heal itself following a severe trauma as it heals for the smaller ones. The eventuality has been called technological immortality. Further, an interesting possibility involves uploading the mind like a computer software on to a new human-body-form generated by cloning, or simply uploading human consciousness onto a computer system, and survive in a virtual environment [36]. Quantum immortality is the name for this kind of development [37]. Other important aspects of current practical scientific thinking about immortality are human cloning, cryonics and nanotechnology. These projections lead us to notions of immortality, a state of non-aging or the unending existence.

\section{Darker-side of life extension}

It appears that with the advancements in medical science, genetics, biotechnology and nanoscience, a true extension of human lifespan will come in the near future [38]. However, it may come at a price and there are fears associated with the extended life.

The tithonus curse or option: fear of old age-tithonus error: In Greek mythology, Tithonus cannot die, but he progressively suffers from all the ill health and frailties of old age. Most of those surviving late into life or Centenarians are the living embodiment of Tithonus' curse. They suffer ill health and frailty associated with old age, are wheelchair or bed-bound, many suffer from dementia, muscle loss, hearing loss, eyesight loss and lack control of their orifices.
The centenarians are not a mystery of nature but old people who happen to suffer the damages of aging a bit longer than others. Like the rest of us, centenarians are governed by the law of mortality, which states that the chances of a person dying double every eight years. With each eight-year period we accumulate more damage generated by the process of metabolism. Initially, during youth, the damage is limited and not apparent because of residual capacity of organs but with age the damage accumulates andante-aging probability of dying from a disease of aging increases exponentially. The centenarians are born with a better genetic constitution helping them to maintain the frail state a little bit longer compared to the average person who lives for about eight decades. These theories enforce the Tithonus Error, which is the belief that extending life means making older people live longer.

Today, most people living in the developed countries can expect to live well into their seventies. Even so, the final years are usually marked by impaired health and often senile dementia. There is a fear that the anti-aging technology may present us with the extended lifespan but limited improvement in quality of life. The nightmare, that we will live longer, but in bad health and mental deterioration, has been called Tithonus Curse or Option - immortal life with subfunctional brain or eternal dementia. But, the goal of anti-aging or rejuvenation science is to offer an opportunity to enable people to be younger for longer, not older for longer, control of aging process and defeat the diseases and disorders related to aging. The aim is not just 'life extension', but and replace it with an extension of healthy life. People will be less likely to commit Tithonus Error if they are presented properly with the intended meaning.

The life on support systems: In the intensive care units, it is commonly seen that for the critically ill patients, more and more invasive procedures are performed to save the life and more and more of vital functions are taken over by bio-machines. Though, the person is considered living till the brain functions, it is an artificially prolonged life on support systems, amounting to a nightmare of proportion of the Tithonus option: a long life with zero quality of life. While genetic engineering and nanotechnology may help in extending the life significantly, it does not follow that future technology will be able to repair all wear-and-tear on the brain and other body organs. If the future technology cannot repair all microscopic injuries, the Tithonus option will result. Considering a poor-quality-of-life better than no life, we may fall in the trap, finally accepting to Tithonus option. People may regard it better than cryopreservation in hope of a novel treatment in the remote future [39].

\section{Positive projections for extended life}

The gursky option: The life extension is projected to be the natural progression of curing diseases by treatment and preventing the effects of aging altogether [40]. The extended life spans will bring positive effects on society of a host of people with the wisdom of 150 years of life and the youthful vitality, which has been called the Gursky option, so named after Ian McDonald's novel, 'The Days of Solomon Gursky', where the inventions allow humans to stave 
off disease and improve their bodies. The issue of overpopulation should not be feared as with spread of benefits of technology, education, and women's rights, fertility rates will decline. Life extension will not place a burden on health care but will itself be associated with good health and disability. The older adults with extended life will be economically productive members of society [41]. The delayed aging will lead to various social changes. The age stratification in the society will disappear, and along with it, many of our current social mores. The new ideas and new possibilities will evolve [42].

\section{Conclusion: From Aging Slowly to Exponential Life Extension and Immortality}

\section{Clarke's third law}

The aging is universal in the kingdom of living. We find people aging; we ourselves age and grow older. There has evolved a whole novel understanding of the biology of aging. The aging is a complex process and affects virtually all organs of the body. A vast body of knowledge can now explain the changes that take place with aging at molecular and cellular level. But, irrational hopes from technology move us away from terra-ferma and are detrimental to rational scientific behavior. Thinking rationally, it is unlikely that something like a pill or potion, can reverse the changes and dysfunction associated with aging. At the same time, the progress in healthcare and technology has made possible to slow aging. Further, there are possibilities of being able to reverse the aging process.

The possibility of a lengthy-healthy life is alluring. As the life expectancy at birth rises and there is taking place an improvement in average and maximum lifespan, the possibility of living life more than never seems logical. The science gives visions; the technology makes the visions possible. The future technology appears to offer us visions that rival the dreams of myth and legend. As per the Arthur C. Clarke's Third Law [43]-'any sufficiently advanced technology is indistinguishable from magic'. One of these magical dreams is that of exponential life extension.

\section{Regenerative medicine and the quest for immortality}

Apart from CR, the regenerative medicine is the next concrete step for achieving longevity. The most promising in regenerative medicine is the therapeutic cloning. A new organ can be grown for transplantation using one's own cells. The process would involve transferring the nucleus from a cell to an enucleated human egg, which would then grow to the blastocyst stage. Stem cells would be harvested from the blastocyst and transformed into the desired tissues for transplant. The regenerative medicine aims higher, it does not retard or slow aging but corrects the organ failure and diseases that accompany aging. It is an advanced form of future science.

Finally, the life is not a myth but an eternal truth. We are because we live. Living a healthy and long life is a common dream. All of us nourish the dream; all of us wish to realize it. Various diseases and infirmity annihilate the dream. Falling prey to aging we lose our health and fitness and pass through an abridged life. Scientifically speaking, the longer life is not separate from aging slowly, rather they are mutually related. With the scientific progress the futuristic visions of achieving significant longevity, if not immortality seems quite possible. Of course, the likelihood of the impact of exponential survival cannot be foreseen in totality [44]. The eternal dream of exponential healthy life extension is on the verge of becoming a reality.

\section{References}

1. Petralia RS, Mattson MP, Yao PJ (2014) Aging and longevity in the simplest animals and the quest for immortality. Ageing Res Rev 16: 66-82.

2. Tosato M, Zamboni V, Ferrini A, Cesari M (2007) The aging process and potential interventions to extend life expectancy. Clin Interv Aging 2(3): 401-412.

3. Strawbridge WJ, Wallhagen MI, Cohen RD (2002) Successful aging and well-being: Self-rated compared with Rowe and Kahn. The Gerontologist 42(6): 727-733.

4. Poulose N, Raghavan R (2014) Aging and injury: Alterations in cellular energetics and organ function. Aging Dis 5(2): 101-08.

5. Gray DA, Woulfe J (2005) Lipofuscin and aging: A matter of toxic waste. Sci Aging Knowl Environ 2005(5): re1.

6. McHugh D (2017) Senescence and aging: Causes, consequences, and therapeutic avenues. J Cell Biology 217(1): 65-77.

7. Gavrilov LA, Gavrilova NS (2001) The reliability theory of aging and longevity. J Theor Biol 213(4): 527-545.

8. Epel ES, Lithgow GJ (2014) Stress biology and aging mechanisms: Toward understanding the deep connection between adaptation to stress and longevity. J Gerontol a Biol Sci Med Sci 69(Suppl 1): S10-S16.

9. Liochev SI (2013) Reactive oxygen species and the free radical theory of aging. Free Radic Biol Med 60: 1-4.

10. Park CB, Larsson NG (2011) Mitochondrial DNA mutations in disease and aging. J Cell Biol 193(5): 809-818.

11. Dato S, Sorensen M, Rango FD, Rose G, Christensen K, et al. (2018) The genetic component of human longevity: New insights from the analysis of pathway-based SNP-SNP interactions. Aging Cell 17(3): e12755.

12. Kerr WG, James J (1994) Gene therapy: current status and future prospects. J Leukoc Biol 56(2): 210-14.

13. Masoro EJ (2000) There takes place an improvement in the immune response, as well restriction and aging: an update. Experimental Gerontology 35: 299-305.

14. Guarente L (2000) Sir2 links chromatin silencing, metabolism, and aging. Genes Dev 14(9): 1021-1026.

15. Guarente L (2013) Calorie restriction and sirtuins revisited. Genes Dev 27(19): 2072-2085.

16. Merkle R (2008) Life, death, and cryonics. Farquhar College of Arts and Sciences Division of Math, Science, and Technology, Nova South-Eastern University, USA.

17. Moen OM (2015) The case for cryonics. Journal of Medical Ethics.

18. Whetstine L, Streat S, Darwin M, Crippen D (2005) Pro/con ethics debate: When is dead really dead? Critical Care 9(6): 538-542.

19. Pichugin Y, Fahy G, Morin R (2006) Cryopreservation of rat hippocampal slices by vitrification. Cryobiology 52(2): 228-240. 
20. McIntyre RL, Fahy GM (2015) Aldehyde-stabilized cryopreservation. Cryobiology 71(3): 448-458.

21. Drexler KE (1987) Engines of creation: The coming era of nanotechnology. Anchor, London, pp. 1-10.

22. Sporns O, Tononi G, Kötter R (2005) The human connectome: A structural description of the human brain. PLoS Comput Biol 1(4): e42.

23. Zador AM, Dubnau J, Oyibo HK, Zhan H, Cao G, et al. (2012) Sequencing the connectome. PLoS Biol 10(10): e1001411.

24. Seung HS (2011) Neuroscience: Towards functional connectomics. Nature 471(7337): 170-172.

25. Seung HS (2013) Connectome: How the brain's wiring makes us who we are. Houghton Mifflin Harcourt, New York, USA.

26. Eth D, Foust J, Whale B (2013) The prospects of whole brain emulation within the next half-century. Journal of Artificial General Intelligence 4(3): 130-152.

27. Human Brain Project.

28. Human Brain Project SP2. Human brain project strategic human brain data.

29. Amunts K, Ebell C, Muller J, Telefont M, Knoll A, et al. (2016) The human brain project: creating a European research infrastructure to decode the human brain. Neuron 92(3): 574-581.

30. Pettit H (2018) First human frozen by cryogenics could be brought back to life 'in just TEN years', claims expert.

31. Marshall J (2006) Life extension research: an analysis of contemporary biological theories and ethical issues. Med Health Care Philos 9(1): 8796.

32. Cerullo MA (2016) The ethics of exponential life extension through brain preservation. Journal of Evolution and Technology 26(1): 94-105.
33. De Grey AD (2003) The foreseeability of real anti-aging medicine: focusing the debate. Exp Gerontol 38(9): 927-934.

34. Norman A, Reedy C (2017) An end to aging: Can science allow humans to become immortal? Future Society. Futurism.

35. Ben B (2000) Immortality: How science is extending your life span-and changing the world. Avon: New York, USA.

36. Alexander B (2010) Rapture: Human immortality and electronic civilization. Publish America.

37. Sandberg A (2013) Feasibility of whole brain emulation. In: Müller VC (Ed.), Theory and philosophy of artificial intelligence, pp. 251-264.

38. Stephen C (2012) Immortality: The quest to live forever and how it drives civilization. Crown.

39. Nikhra V (2006) Ageing slowly, Living longer. Spandan Innovative in association with Sahni Publications. ISBN-81-7564-371-374.

40. De Grey A, Rae M (2008) Ending aging: The rejuvenation breakthroughs that could reverse human aging in our lifetime. St. Martin's Griffin.

41. Zhavoronkov A (2013) The ageless generation: How advances in biomedicine will transform the global economy. Palgrave Macmillan, New York, USA.

42. De Grey AD (2005) Life extension, human rights, and the rational refinement of repugnance. Journal of Medical Ethics 31(11): 659-663.

43. Arthur CC (2000) Profiles of the future: An inquiry into the limits of the possible. Publisher: Phoenix, The Orion Publishing Group Ltd.

44. Friebe M (2017) Exponential technologies+reverse innovation=solution for future healthcare issues? what does it mean for university education and entrepreneurial opportunities? OJBM 5(3): 458-469,
Creative Commons Attribution 4.0

International License

For possible submissions Click Here

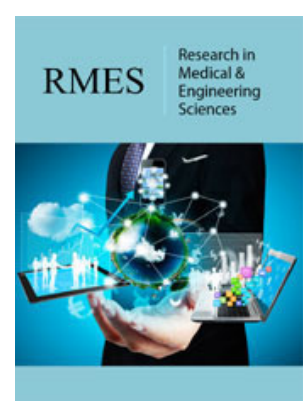

Research in Medical \& Engineering Sciences

\section{Benefits of Publishing with us}

- High-level peer review and editorial services

- Freely accessible online immediately upon publication

- Authors retain the copyright to their work

- Licensing it under a Creative Commons license

- Visibility through different online platforms 